\title{
Spatial-temporal analysis of prostate cancer incidence from the Pennsylvania Cancer Registry, 2000-2011
}

\author{
Ming Wang, ${ }^{1}$ Stephen A. Matthews, ${ }^{2,3}$ Khaled Iskandarani, ${ }^{1}$ Yimei Li, ${ }^{4}$ Zheng Li, ${ }^{1}$ \\ Vernon M. Chinchilli, ${ }^{1}$ Lijun Zhang ${ }^{5}$ \\ ${ }^{1}$ Department of Public Health Sciences, Pennsylvania State University, Hershey, PA; ${ }^{2}$ Department of \\ Sociology, Pennsylvania State University, University Park, PA; ${ }^{3}$ Department of Anthropology, Pennsylvania \\ State University, University Park, PA; ${ }^{4}$ Department of Biostatistics, University of Pennsylvania, \\ Philadelphia, PA; ${ }^{5}$ Institute for Personalized Medicine, Pennsylvania State University, Hershey, PA, USA
}

\begin{abstract}
Prostate cancer is the most common cancer diagnosed among males, and the incidence in Pennsylvania, USA is considerably higher than nationally. Knowledge of regional differences and
\end{abstract}

Correspondence: Ming Wang, Division of Biostatistics and Bioinformatics, Department of Public Health Sciences, Penn State University College of Medicine, 90 Hope Drive, Hershey, PA 170330850, USA.

Tel: +1-717-531-5745 - Fax: +1-717-531-5779.

E-mail: mwang@phs.psu.edu

Key words: Prostate cancer; Spatial-temporal analysis; Incidence rate; Hierarchical generalised linear models; USA.

Contributions: MW and SAM contributed equally.

Acknowledgements: we thank Peter Imrey and Eugene J. Lengerich for helpful comments for this manuscript, and James Rubertone for data extraction and other inquiries related to the Pennsylvania Cancer Registry. Dr. Wang's research was supported by the National Center for Advancing Translational Sciences, Grant 5 UL1 RR0330184-04, KL2 TR000126 and TR002015. The content is solely the responsibility of the authors and does not necessarily represent the official views of the NIH.

Ethical approval: upon the request by the Pennsylvania Department of Health, the application for protected data access has been approved, and this project also obtained IRB approval from Penn State University (http://irb.psu.edu).

Received for publication: 2 August 2017.

Revision received: 7 November 2017.

Accepted for publication: 9 November 2017.

CC Copyright M. Wang et al., 2017

Licensee PAGEPress, Italy

Geospatial Health 2017; 12:611

doi:10.4081/gh.2017.611

This article is distributed under the terms of the Creative Commons Attribution Noncommercial License (CC BY-NC 4.0) which permits any noncommercial use, distribution, and reproduction in any medium, provided the original author(s) and source are credited. time trends in prostate cancer incidence may contribute to a better understanding of aetiologic factors and racial disparities in outcomes, and to improvements in preventive intervention and screening efforts. We used Pennsylvania Cancer Registry data on reported prostate cancer diagnoses between 2000 and 2011 to study the regional distribution and temporal trends of prostate cancer incidence in both Pennsylvania White males and Philadelphia metropolitan area Black males. For White males, we generated and mapped county-specific age-adjusted incidence and standardised incidence ratios by period cohort, and identified spatial autocorrelation and local clusters. In addition, we fitted Bayesian hierarchical generalised linear Poisson models to describe the temporal and aging effects separately in Whites state-wide and metropolitan Philadelphia blacks. Incidences of prostate cancer among white males declined from 2000-2002 to 2009-2011 with an increasing trend to some extent in the period 2006-2008 and significant variation across geographic regions, but less variation exists for metropolitan Philadelphia including majority of Black patients. No significant aging effect was detected for White and Black men, and the peak age group for prostate cancer risk varied by race. Future research should seek to identify potential social and environmental risk factors associated with geographical/racial disparities in prostate cancer. As such, there is a need for more effective surveillance so as to detect, reduce and control the cancer burden associated with prostate cancer.

\section{Introduction}

Prostate cancer screening rates have increased in recent decades rendering the disease the most commonly diagnosed cancer among American men, with treatment costing approximately \$14-15 billion in 2015 (Farwell et al., 2007; Jemal et al., 2010). In 2013, Pennsylvania ranked third among states in annual prostate cancer incidence (132.2 cases per 100,000 men) and sixth in cancer mortality (Siegel et al., 2012). Indeed, it is estimated that over one-sixth of Pennsylvania men will be diagnosed with prostate cancer during their lifetimes (Pennsylvania Prostate Cancer Coalition; http://www.paprostatecancer.org/prostate-cancer/). While the age-adjusted incidence rate of prostate cancer in Pennsylvania declined between 2001 and 2012, the pace of decline has been unstable and the temporal trend deserves more attention (Reese et al., 2016). 
The extant literature has shown that prostate cancer occurs more often in African American men compared to White men (Consedine et al., 2006; Reese et al., 2016). A 2006 special issue of the American Journal of Preventive Medicine (30:2) on this subject indicated that prostate cancer incidence and progression vary widely by geographic location (Mather et al., 2006). Also, the identification of risk factors to effectively reduce prostate cancer incidence, morbidity and mortality remains a serious public health challenge (Giovannucci et al., 2007; Patel and Klein, 2009). Therefore, the joint examination of racial and geographical disparities for prostate cancer in Pennsylvania and investigation of the potential risk factors are of both scientific and policy interest.

Mapping the geographic variation in prostate cancer incidence can be useful for developing effective cancer control and prevention programmes, as well as for generating etiological hypotheses (e.g., associated with the age or race structure of geographic areas) (Kulldorff et al., 2006; Richards and Rushton, 2006). Mapping, while useful, requires accompanying statistical analyses to reduce the influence of chance variation for valid inference; however, the majority of the current work commonly only adopts simple summary statistics or epidemiological approaches, so application of advanced statistical modelling for analyses is usually lacking. Examples include studies at the county-level for the U.S. for the period 1968-1998 (Rogerson et al., 2006) and for selected states [Virginia 1990-1999 by Oliver et al. (2006); Louisiana 1988-1999 by Mather et al. (2006); and 2007-2010 by Onicescu et al. (2015) and Pennsylvania 1992-2012 by Reese et al. (2016)] have examined changes in either the spatial or the temporal pattern of prostate cancer incidence or mortality. In addition, some studies relied on cross-sectional approaches for risk factor identification (Patel and Klein, 2009).

The objective of this paper was to utilise rigorous analytical tools, together with longitudinal geo-spatial prostate cancer registry data (2000-2011) to explore regional and temporal variations in incidence rates among White men in Pennsylvania and further to estimate the effects of time and aging risk factors and race-specific disparities. In doing so, we seek to identify the quantitative effects of aging and diagnosis time period and to examine racial differences (Waller et al., 1997; Jin and Carlin, 2005).

\section{Materials and Methods}

\section{Data}

All reported prostate cancer diagnoses and deaths between years 2000-2011 were obtained from the Pennsylvania Cancer Registry (PCR) (http://www.health.pa.gov). The PCR began collecting annual data in 1982, with individual-level reports obtained from hospitals, pathology laboratories, radiation treatment centres, medical oncology centres, physician offices, nursing homes, autopsy reports, death certificates and other hospital out-patient units and surgery centres across Pennsylvania, including complete vital status and selected exploratory factors. The dataset was deidentified, processed and assembled for analysis. After PCR data processing, our analytical sample for the spatial-temporal analysis included a total of 119,968 cases.

For each individual case, the residential location at diagnosis was assigned to a county Federal Information Processing Standards (FIPS) code number. All maps were generated in ArcGIS 10.1 (ESRI, Redlands, CA, USA). To achieve relatively stable estimates for investigating the spatial-temporal trend, we aggregated the twelve years of data into four time periods, each diagnosis period covering three years (i.e. 2000-2002, 2003-2005, 2006-2008, and 2009-2011). We used six age groups (i.e. $\leq 44,45-$ $54,55-64,65-74,75-84$, and $\geq 85$ ) and for race, we focused on White men for a state-wide analysis, and Black men in the Pennsylvania metropolitan area.

The annual estimates of the county resident populations by age group and race for the years 2000-2011 were based on the U.S. Census Bureau's Population Estimates Program. We used the 2011 Census as well as 2000-2010 inter-Censal revisions. The dataset is available from the U.S. Census Bureau (https://www. census.gov/topics/income-poverty/income/data/datasets.html).

\section{Statistical methods}

By linking the PCR and U.S. Census Bureau estimates, the crude and age-adjusted prostate cancer incidence rates (per 100,000 men-at-risk) were obtained for each year, and the incidence rates by time period (defined above) were calculated as the average. Specifically, the age-adjusted incidence rates were calculated based on the weighted sum of the products by multiplying the age-specific incidence rate and the proportion of the standard population in that age group. Furthermore, indirect standardised incidence ratios (SIRs) were calculated as the ratios of the observed number of cancer cases to the expected number multiplied by 100 , where the state-wide age-specific incidence rates pooled over the twelve-year period was used as an internal standard to calculate the expected number (following the work by Mather et al., 2006). In addition empirical Bayesian smoothing estimates of SIRs were also examined.

The global Moran's I was used for significant global autocorrelation testing for each time period, and the local Moran tests helped identify local outliers which potentially contribute to the global statistic using unsmoothed SIRs. With regard to the weight matrix, we defined contiguity as counties sharing more than one boundary point and utilised binary weights (Waller and Gotway, 2004). The maps were transformed using the Lambert Conformal Conic projection and the Euclidean distances for spatial neighbour links were calculated.

Bayesian hierarchical generalised linear modelling (HGLM) with a Poisson distribution was fitted, incorporating spatial correlation, where a localised conditionally autoregressive (CAR) model was applied for spatial random effects based on dissimilarity metrics with binary neighbourhoods (Lee and Mitchell, 2012; Lee et al., 2014). This model was fitted first for White men across Pennsylvania, and then for Black men using a sub-region equivalent to the Philadelphia metropolitan area. The parameter estimation was based on the Markov Chain Monte Carlo (MCMC) algorithm with a combination of Gibbs sampling and MetropolisHastings steps. The fitted model was given by:

$\log \left(Y_{i}\right)=X_{i}^{T} \beta+\mu_{i}+\varepsilon_{i}+\log \left(E_{i}\right)$

where $Y_{i}$ and $E_{i}$ are the observed and expected numbers of cases for the $i^{\text {th }}$ subgroup (determined by the age group and diagnosis period) and $X_{i}$ the vector of covariates of interest (i.e. age group and diagnosis period). The distributional assumptions were that the random effect $\mu_{i} \sim N\left(0, \sigma_{\varepsilon}^{2}\right)$, the error term $\varepsilon_{i} \sim N\left(0, \sigma_{\varepsilon}^{2}\right)$, and their independence $\mu_{i} \perp \varepsilon_{\mathrm{i}}$ The function S.CARdissimilarity in the R package CARBayes was applied for this model fitting. In particular, 10,000 MCMC samples were generated with 2,000 samples discarded as the burn-in period and the level of thinning set at 100 . 


\section{Results}

The total dataset in this study included a majority of White men, with the percent representing Black men across all years amounting to $10.33 \%$ (with $9.89 \%$ in $2000-2002$ and $10.56 \%$ in $2009-2011$ ). The descriptive statistics of all variables are shown in Table 1 . The distributions of the total number of cases across all four periods were found to be relatively stable (roughly 30,000 in each three-year period). The distributions of cases by age group between White and Black men revealed considerable heterogeneity $(\mathrm{P}<0.05)$. Black men comprised $23.76 \%$ of the prostate cancer cases among those aged $\leq 44$, over $16 \%$ of the cases in Pennsylvania men for the $45-54$ age group, and approximately $12 \%$ in the 55-64 age group. The black population in Pennsylvania resides predominantly in metropolitan areas, in particular in the Philadelphia metropolitan area counties (approximately half of all black men in Pennsylvania live in just one county, i.e. Philadelphia County, one of the five included in the Philadelphia Metro area). Overall, $52 \%$ of all prostate cancer cases during the years 2000-2011 were located in Pennsylvania's two largest metropolitan statistical areas (MSAs), i.e. Philadelphia MSA and Pittsburgh MSA. In comparison, over $86 \%$ of all Black cases in Pennsylvania $(10,700$ of 12,397$)$ were observed in these two MSAs, with Philadelphia MSA alone accounting for over $71 \%$ of the state total. Of note, in the Philadelphia MSA, the number of Black cases amounted to almost a quarter of all cases reported $(8,895$ of 38,512). To reinforce the spatial concentration of prostate cancer cases found among Black men, less than $7 \%$ of all black cases ( 814 cases across 12 years) were from Pennsylvania's non-metropolitan counties.

\section{Incidence estimation}

There was significant variation across geographic regions (county level) and over time (periods) in prostate cancer incidence for White men in Pennsylvania. Let us begin with a map of the ageadjusted incidence rates of prostate cancer among White men across four time periods, followed by the SIR maps. The ageadjusted incidence rates per 100,000 white men are shown in Figure 1. For ease of interpretation, the maps have been designed with time-constant legend classes. The temporal trends revealed an overall decline across the four time periods for White men with an increase in rates observed for the years 2006-2008 (Table 1). While a considerable heterogeneity in prostate cancer incidence rates across the state was found, during the earliest time period (20002002), the western half of the state tended to have the highest rates for White men (Figure 1), with the very highest rates found in counties along the southern border with Maryland (e.g., Bedford and Somerset counties) and counties immediately to the north (e.g., Cambria County). These three counties, Bedford, Cambria and Somerset, also maintained the highest incidence rates in the following time period (2003-2005). As previously noted, the increase in prostate cancer incidence was a feature of the years 2006-2008, with relatively high and homogenous rates for White men observed across much of Western Pennsylvania. By the period 2009-2011, the incidence rates of prostate cancer among White men diminished relative to the years 2000-2002, with the highest rates found in some of the smaller western tier counties (i.e. Mercer and Butler counties) and also Bedford county. Comparing

Table 1. Descriptive statistics for prostate cancer in Pennsylvania for the period 2000-2011.

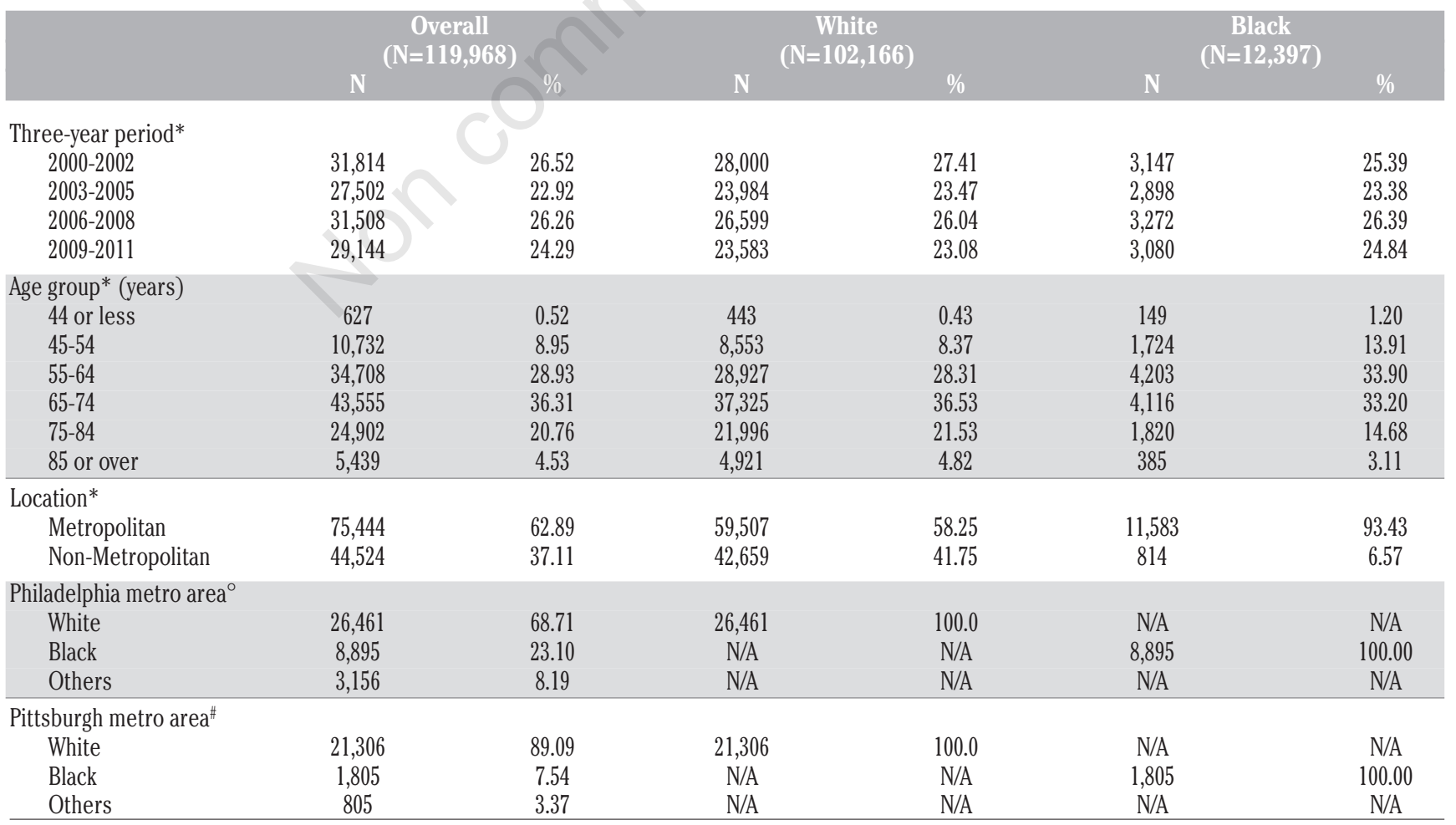

N/A, not available. ${ }^{\circ}$ Bucks, Chester, Delaware, Montgomery and Philadelphia counties; For this area, 2,289 for 2000-2002, 2,114 for 2003-2005, 2,363 for 2006-2008 and 2,129 for 2009-2011; also, 109 for age $\leq 44,1,169$ for

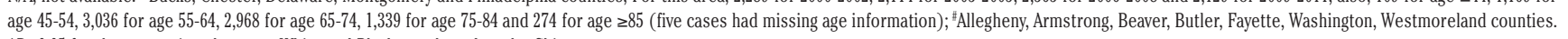
${ }^{*} \mathrm{P}<0.05$ for the comparison between White and Black men based on the Chi-square tests. 
the two most populous counties, the incidence rate of prostate cancer among White men in Philadelphia County (i.e. Philadelphia) exceeded that for Allegheny County (i.e. Pittsburgh) in all fourtime periods.

Figure 2 provides the SIR maps for White men across the four time periods based on the observed and expected number of cases; the expected numbers were calculated for each year and the average age-adjusted incidence rates across the entire time-period. The counties with higher (lower) SIRs than expected corresponded to a higher (lower) incidence of prostate cancer. While the overall trend in the pattern for White men seemed to be in decline over time, with the smallest SIRs for the years 2009-2011, the heterogeneity in SIRs across and within counties over time was relatively high. That is, while SIRs appeared to decrease over time and seemed fairly homogenous across the counties, especially after 2006, the highest SIR in Elk County was double the lowest rates in Pennsylvania. Also, to account for potential heterogeneity, the smoothed SIRs for White men based on the empirical Bayesian approach were also investigated (not shown here) leading to similar conclusions. In summary, a general decline was observed across the 12-year window with growth in 2006-2008 followed by a resumption of the decline in 2009-2011. In addition, the prostate cancer incidences among White men in several central and southern counties declined significantly over the 12-year time period.

\section{Spatial autocorrelation}

The global Moran's I statistics with 95\% confidence intervals
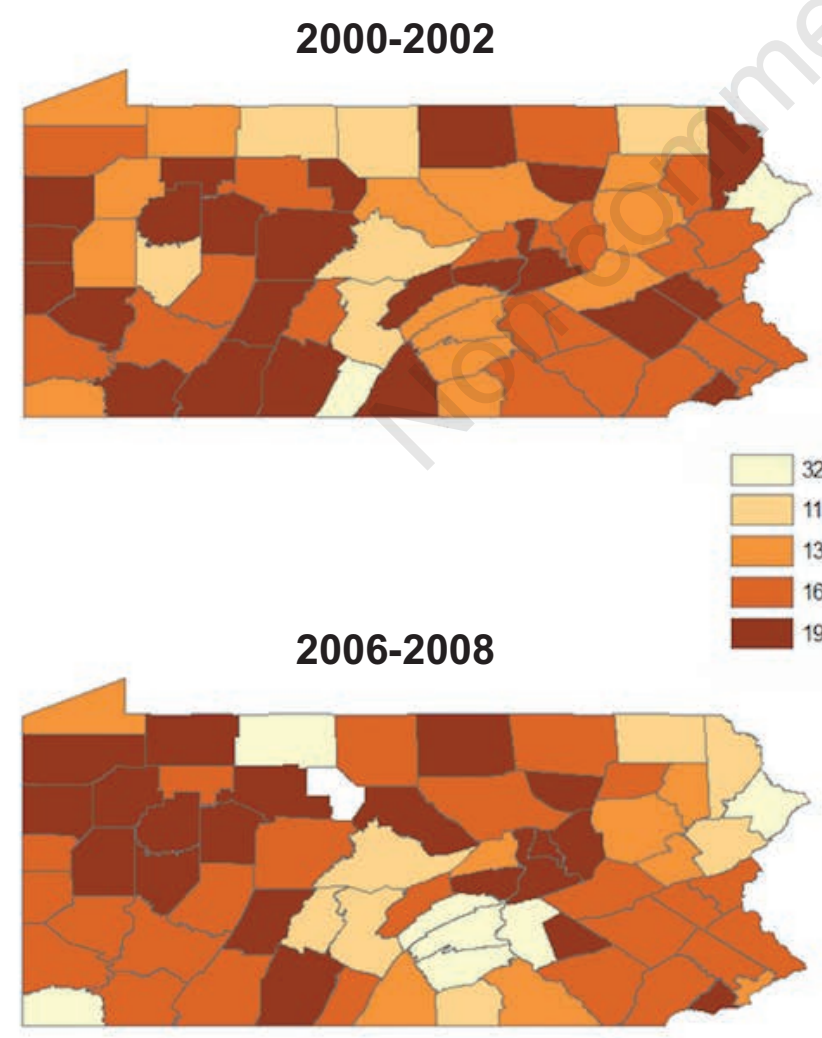

(CI) were calculated for each of the four time periods using three different spatial weights, row-standardised, binary and inverse distance weights (not shown here). Irrespective of the spatial weights used, however, the results were similar across the time periods, with negative spatial autocorrelation estimates (e.g., -0.014 with $95 \%$ CI $[-0.159,0.131]$ for $2000-2002$ with binary weights) indicating a very modest dissimilarity across counties though these were not significant. This lack of evidence for high spatial structure indicates, as is partly visible in the maps (Figure 2), the considerable heterogeneity across counties, including variability among_near neighbours. The local Moran's I scatterplots for White men based on age-adjusted SIRs were also checked. The local indicator of spatial autocorrelation (LISA) identified significant clusters of counties with either high or low age-adjusted SIRs (e.g., HH or high-high and LL or low-low) and clusters where the focal county and its neighbours differed significantly, e.g., where a focal county has a high $(\mathrm{H})$ age-adjusted SIR and adjacent counties low (L) age-adjusted SIRs, which is defined as a high-low (HL) cluster; or the opposite situation, which results in a low-high (LH) cluster. The LISA cluster maps with influential outliers are presented in Figure 3 (None means not significant here). Note that for White men, there exist variability in the location of LISA clusters over the time periods examined.

\section{Bayesian hierarchical generalised linear modelling}

In this section we present the results of a hierarchical generalised linear modelling (HGLM) model controlling for the covari-

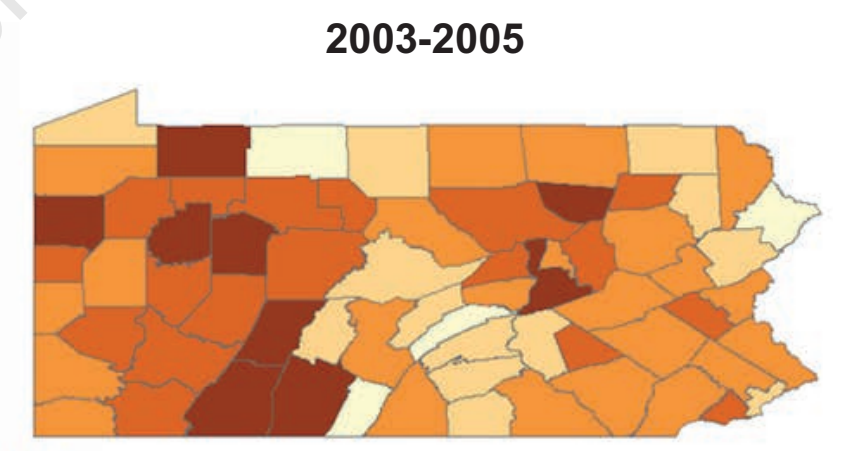

$32.8-111.0$
$11.1-138.0$
$38.1-162.0$
$62.1-196.0$
$96.1-266.0$

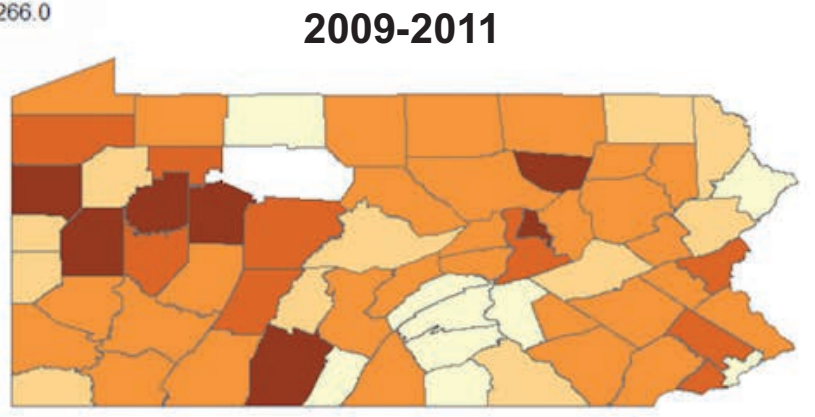

Figure 1. Age-adjusted incidence (per 100,000 White men at risk) by county for White men. 
ates year groupings of prostate cancer diagnosis and age groups (Table 2). The results for White men (top panel) show that compared with the age group $\leq 44$ (the reference group) all other age groups have higher incidence rates though these differences are not statistically significant; those aged 55-64 have the highest risk for prostate cancer. Consistent with earlier results when we examined time periods (with the period 2000-2002 as the reference category), we found that White men have significantly lower incidence rates in the later periods, with the last period (2009-2011) having the smallest incidence. Table 2 includes an exploration based on incidence data for Black men in the Philadelphia metropolitan area (bottom panel). The results showed a similar temporal pattern of incidence as among White men across the state; that is, the time period is significantly associated with prostate cancer incidence rates for Black men; however, among all age groups, Black men aged 45-54 have the highest rates immediately followed by those aged 75-84. Those aged 85 or more have the lowest risk, which may be due to competing deaths.

\section{Discussion}

This paper provides a descriptive account of the spatial and temporal variability in prostate cancer incidence over a 12-year period (2000-2011) for White men in Pennsylvania and Black men in the Philadelphia metropolitan area. Prostate cancer kills thousands of Pennsylvania men each year and a spatio-temporal perspective that can reveal regional and local patterns and may pro-
Table 2. Results for Bayesian hierarchical generalised linear modelling for White men state-wide and Black men in the Philadelphia Metro area.

\begin{tabular}{lccc} 
& Median & $2.5 \%$ & $97.5 \%$ \\
White & & & \\
Intercept & 0.106 & -0.054 & 0.264 \\
Age group (years) & & & \\
44 or less & Reference & & \\
45-54 & 0.043 & -0.113 & 0.206 \\
$55-64$ & 0.047 & -0.108 & 0.208 \\
$65-74$ & 0.042 & -0.114 & 0.211 \\
$75-84$ & 0.042 & -0.116 & 0.212 \\
85 or over & 0.021 & -0.148 & 0.200 \\
Period cohort & & & \\
2000-2002 & Reference & & \\
$2003-2005$ & -0.189 & -0.221 & -0.159 \\
$2006-2008$ & -0.129 & -0.156 & -0.102 \\
$2009-2011$ & -0.305 & -0.336 & -0.277 \\
Black* & & & \\
Intercept & 0.106 & -0.211 & 0.323 \\
Age group (years) & Reference & & \\
44 or less & 0.012 & -0.235 & 0.326 \\
45-54 & -0.001 & -0.224 & 0.327 \\
$55-64$ & -0.005 & -0.233 & 0.310 \\
65-74 & 0.005 & -0.217 & 0.315 \\
$75-84$ & -0.025 & -0.324 & 0.356 \\
85 or over & Reference & & \\
Period cohort & -0.127 & -0.229 & -0.023 \\
2000-2002 & -0.074 & -0.165 & -0.013 \\
2003-2005 & -0.251 & -0.351 & -0.142 \\
2006-2008 & & \\
2009-2011 & & & \\
\hline
\end{tabular}

*The model for Black men based on the sub-region data from Philadelphia Metro area.
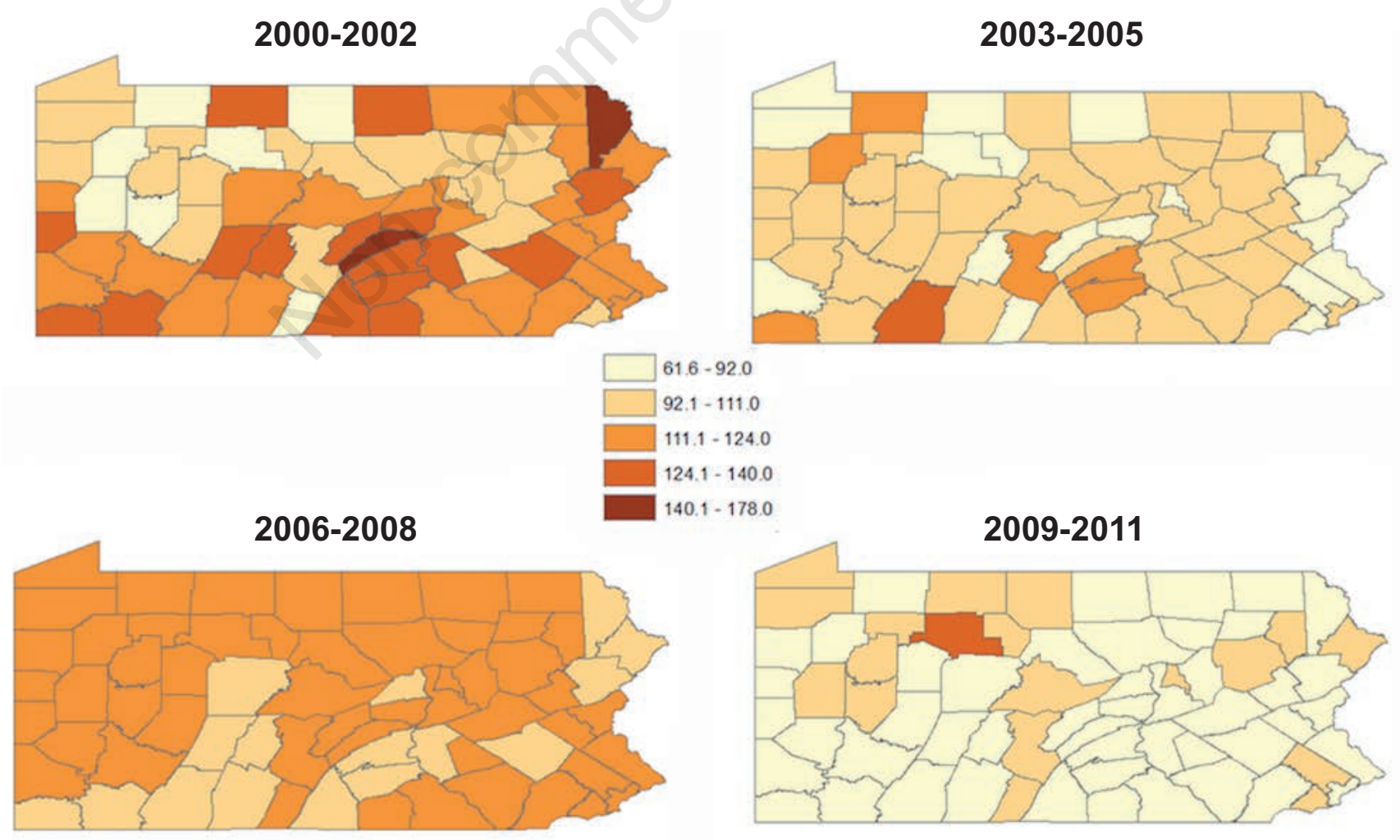

$61.6-92.0$

$92.1-111.0$

$111.1-124.0$

$124.1-140.0$

$140.1-178.0$

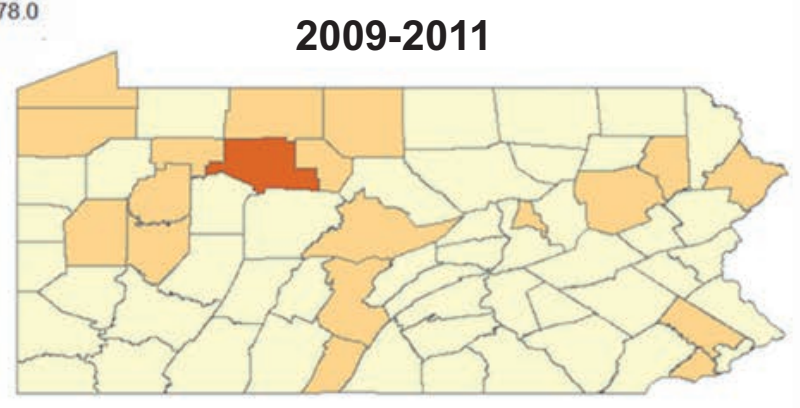

Figure 2. Standardised incidence ratios (multiplied by 100) by county for White men. 
vide insights that can inform comprehensive cancer control strategies that seek to minimise the cancer burden and disparities associated with this form of cancer. This is especially important in a state such as Pennsylvania where the population is aging, mostly the result of aging in place, and a high percent (and a large number) of the population live in non-metropolitan counties. Approximately three-quarters of the population of the state were born in Pennsylvania and currently the state ranks the fourth highest in the proportion of elderly (U.S. Census Bureau, 2010; Pennsylvania Facts, 2014) The size and age composition of the population can challenge the delivery of preventive and treatment services and as well as patient concordance with suggested cancer treatment.

The general trend in prostate cancer incidence in Pennsylvania has been declining since the millennium shift. However a more detailed temporal and spatial breakdown reveals that the pace of decline was not continuous, with elevated rates observed during the years 2006-2008, and that the prostate cancer rates varied widely even within this one state. These findings are consistent with previous literature (Siegel et al., 2012; Mamula, 2013). Using SIRs and smoothed SIRs, we also identify counties where the prostate cancer rates seemed to follow a trend counter to that of the state. That is, a small number of counties experienced higher prostate cancer rates among White men in the latest period investigated (2006-2011) than at the beginning of our temporal coverage (2000-2005). The counties that differed from the state trend represent local areas in which a more detailed epidemiological study of prostate cancer among White men and potential social and environmental risk factors might be focused.

The Black population in Pennsylvania is highly concentrated in the Philadelphia metropolitan area, and for the most part our

Period 2000-2002

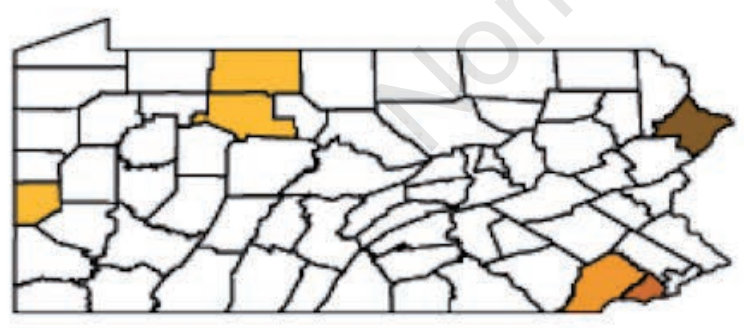

Period 2006-2008

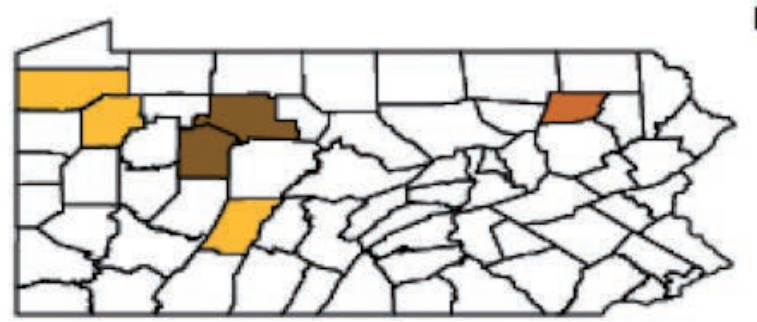

analysis finds similar rates and declines in rates as with the statewide study of White men. That said, descriptive data reveal that the incidence rates among Black men less than 55 years old in Pennsylvania is disproportionately high compared to similar age groups of White men. Indeed, $50 \%$ of all Black cases occurred in men less than 65 compared to just $37 \%$ of all White cases in this age group. Coupled with the spatial concentration of Black men in the largest metropolitan counties (i.e. Philadelphia and Pittsburgh) implies the need for a stronger focus on prostate cancer in these parts of the state. The high incidence rates among Black men certainly raises important questions regarding both the provisioning and the uptake of prostate cancer screening within the large metropolitan areas but also in some of the smaller metropolitan areas where the Black population is slowly growing (e.g., Harrisburg, Dauphin County). That is, we know that prostate cancer occurs more often in Black men and researchers need to pay more attention to the locations where Black men live. Indeed, this focus is especially important because the mortality rate ratio between Black and White men is higher for prostate cancer than for any other cancer (DeSantis et al., 2013). Though a recent study suggested that prostate cancer screening explained $45-70 \%$ of the observed decline in prostate cancer mortality in the U.S. between years 1980-2000 (Jemal et al., 2002; Etzioni et al., 2008), the clinical evidence for the efficacy of prostate cancer screening remains mixed (Andriole et al., 2009; Schroder et al., 2009).

There are limitations to this study. The race and ethnic composition of the state of Pennsylvania, specifically the spatial concentration of Blacks in the largest metropolitan areas (notably Philadelphia, Pittsburgh and Harrisburg), prevents an analysis examining the racial disparities in prostate cancer incidence across the whole state. It is also worth noting that the focus on one state,

Figure 3. The local indicator of spatial autocorrelation cluster maps for White men. 
in this case Pennsylvania, limited activities to examination of prostate cancer rates within the boundaries of this state ignoring edge effects and influences on such data from surrounding states.

\section{Conclusions}

Our analysis detected spatio-temporal variation in prostate cancer incidence rates among White men in the state of Pennsylvania between 2000 and 2011. Specific counties fair less well when comparing absolute rates as well as the pace and persistence of decline in prostate cancer among White men. Our analysis confirms the variability in the peak age for prostate cancer by race (White vs Black men) and, coupled with the spatial concentration of Black men in Philadelphia, indicates a need for more effective surveillance of this population and continued efforts to minimise prostate cancer burdens and disparities. Both patterns hint at possibilities for more detailed epidemiological studies that could examine sets of social determinants and environmental factors associated with prostate cancer.

\section{References}

Andriole GL, Crawford ED, Grubb RL, 3rd, Buys SS, Chia D, Church TR, Fouad MN, Gelmann EP, Kvale PA, Reding DJ, Weissfeld JL, Yokochi LA, O’Brien B, Clapp JD, Rathmell JM, Riley TL, Hayes RB, Kramer BS, Izmirlian G, Miller AB, Pinsky PF, Prorok PC, Gohagan JK, Berg CD, Team PP, 2009. Mortality results from a randomized prostate-cancer screening trial. New Engl J Med 360:1310-9.

Consedine NS, Morgenstern AH, Kudadjie-Gyamfi E, Magai C, Neugut AI, 2006. Prostate cancer screening behavior in men from seven ethnic groups: the fear factor. Cancer Epidemiol Biomarkers Prev 15:228-37.

DeSantis C, Naishadham D, Jemal A, 2013. Cancer statistics for African Americans, 2013. CA Cancer J Clin 63:151-66.

Etzioni R, Tsodikov A, Mariotto A, Szabo A, Falcon S, Wegelin J, DiTommaso D, Karnofski K, Gulati R, Penson DF, Feuer E, 2008. Quantifying the role of PSA screening in the US prostate cancer mortality decline. Cancer Causes Control 19:175-81.

Farwell WR, Linder JA, Jha AK, 2007. Trends in prostate-specific antigen testing from 1995 through 2004. Arch Intern Med 167:2497-502.

Giovannucci E, Liu Y, Platz EA, Stampfer MJ, Willett WC, 2007. Risk factors for prostate cancer incidence and progression in the health professionals follow-up study. Int $\mathrm{J}$ Cancer 121:1571-8.

Jemal A, Kulldorff M, Devesa SS, Hayes RB, Fraumeni JF, 2002. A geographic analysis of prostate cancer mortality in the United States, 1970-89. Int J Cancer 101:168-74.
Jemal A, Siegel R, Xu J, Ward E, 2010. Cancer statistics, 2010. CA Cancer J Clin 60:277-300.

Jin X, Carlin BP, 2005. Multivariate parametric spatiotemporal models for county level breast cancer survival data. Lifetime Data Anal 11:5-27.

Kulldorff M, Song C, Gregorio D, Samociuk H, DeChello L, 2006. Cancer map patterns: are they random or not? Am J Prev Med 30:37-49.

Lee D, Mitchell R, 2012. Boundary detection in disease mapping studies. Biostatistics 13:415-26.

Lee D, Rushworth A, Sahu SK, 2014. A Bayesian localized conditional autoregressive model for estimating the health effects of air pollution. Biometrics 70:419-29.

Mamula KB, 2013. PA ranks third in cancer incidence. Available from: http://www.bizjournals.com/pittsburgh/news/2013/ 10/29/pa-ranks-fifth-in-cancer-incidence.html

Mather FJ, Chen VW, Morgan LH, Correa CN, Shaffer JG, Srivastav SK, Rice JC, Blount G, Swalm CM, Wu X, Scribner RA, 2006. Hierarchical modeling and other spatial analyses in prostate cancer incidence data. Am J Prev Med 30:88-100.

Oliver MN, Smith E, Siadaty M, Hauck FR, Pickle LW, 2006. Spatial analysis of prostate cancer incidence and race in Virginia, 1990-1999. Am J Prev Med 30:67-76.

Onicescu G, Lawson A, Zhang J, Gebregziabher M, Wallace K, Eberth JM, 2015. Bayesian accelerated failure time model for space-time dependency in a geographically augmented survival model. Stat Methods Med Res 26:2244-56.

Patel AR, Klein EA, 2009. Risk factors for prostate cancer. Nat Clin Pract Urol 6:87-95.

Reese AC, Wessel SR, Fisher SG, Mydlo JH, 2016. Evidence of prostate cancer "reverse stage migration" toward more advanced disease at diagnosis: Data from the Pennsylvania Cancer Registry. Urol Oncol 34:335.e21-28.

Richards TPL, Rushton G, 2006. Prostate cancer and geographic information systems (GIS). Am J Prev Med 30:2.

Rogerson PA, Sinha G, Han D, 2006. Recent changes in the spatial pattern of prostate cancer in the U.S. Am J Prev Med 30:50-9.

Schroder FH, Hugosson J, Roobol MJ, Tammela TL, Ciatto S, Nelen V, Kwiatkowski M, Lujan M, Lilja H, Zappa M, Denis LJ, Recker F, Berenguer A, Maattanen L, Bangma CH, Aus G, Villers A, Rebillard X, van der Kwast T, Blijenberg BG, Moss SM, de Koning HJ, Auvinen A, Investigators E, 2009. Screening and prostate-cancer mortality in a randomized European study. New Engl J Med 360:1320-8.

Siegel R, Naishadham D, Jemal A, 2012. Cancer statistics, 2012. CA Cancer J Clin 62:10-29.

Waller LA, Carlin BP, Xia H, Gelfand AE, 1997. Hierarchical spatio-temporal mapping of disease rates. J Am Stat Assoc 92:607-17.

Waller LA, Gotway CA, 2004. Applied spatial statistics for public health data. John Wiley \& Sons, Hoboken, NJ, USA. 